\title{
Summary of the Prevention of Catheter-Associated Urinary Tract Infection in An Intensive Care Unit
}

\section{Waluyo, Yulia Indah Permata Sari, Ulfa Nur Rohmah, Saskiyanti Ari Andini, and Hendrik Prayitno Luwao}

Faculty of Nursing, Universitas Airlangga, Surabaya, Indonesia

\section{ABSTRACT}

Background: Catheter-associated urinary tract infection is the most common type of nosocomial infection in an intensive care unit. The aim of this study was to examine the existing evidence of preventative measures against catheterassociated urinary tract infection being implemented to reduce urinary tract infection in intensive care units.

Method: Databases were used to identify potential articles, namely Scopus, Pubmed, EBSCO and Proquest, limited to those published within the last 5 years from 2013 to 2018. The literature review used the keyword prevention, CAUTI and ICU. In the article search using "AND", only 14 studies met the inclusion criteria. Across the 14 studies, 42486 participants and a mean 3540 per trial were included.

Discussion: Nursing round, CAUTI bundle, bacitracin and cranberry, Nursedriven protocol, protocol by team/ developmental protocol, surveillance of CAUTI, education, performance feedback, and general cultural practices alongside the American College of Critical Care Medicine and the Infectious Disease Society of America present guidelines that recommend CAUTI preventive practices that can be implemented to reduce the incidence of CAUTI in ICUs.

Conclusion: From the several strategies used to prevent CAUTI, the most effective is the multidimensional approach because this approach combines several interventions and it also involves other practitioners. A multidimensional approach is more effective than a single dimensional approach in ICU.
\end{abstract}

\section{ARTICLE HISTORY}

Received: Dec 26, 2019

Accepted: Dec 31, 2019

\section{KEYWORDS}

CAUTI; ICU; nosocomial infection; multidimensional approach

\section{CONTACT}

Waluyo

$\triangle$ waluyo-2018@fkp.unair.ac.id $\equiv$ Faculty of Nursing, Universitas Airlangga, Surabaya, Indonesia

Cite this as: Waluyo, W. L, Sari, I. P, Rohmah, U. N, Andini, S. A, and Luwao, H. P. (2019). Summary of the Prevention of Catheter-Associated Urinary Tract Infection in An Intensive Care Unit. Jurnal Ners, 14(3si), 103-107. doi:http://dx.doi.org/10.20473/jn.v14i1.17010

\section{INTRODUCTION}

Catheter-associated urinary tract infections (CAUTIs) are among the most common device-associated healthcare-acquired infections in intensive care units (ICUs)(Navoa-Ng et al., 2013). CAUTIs are responsible for prolonged hospital lengths of stay, bacterial resistance, morbidity and increased healthcare costs(Navoa-Ng et al., 2013). If CAUTI is not immediately treated, it will cause urosepsis and can even cause death. It is estimated that $17 \%-69 \%$ of CAUTI can be prevented by applying infection prevention practices based on proven evidence. This means that the 380,000 infections and 9,000 deaths associated with CAUTI every year can be prevented by implementing this(Gould, Umscheid, Agarwal,
Kuntz, \& Pegues, 2017). CAUTIs increase the cost of treatment and length of stay. There is growing concern over the health care cost, especially in terms of preventable problems(Underwood, 2015).

CAUTI prevention efforts have been implemented in healthcare facilities and ICUs globally. However, standardization of the interventions and protocols used is lacking. A review of the literature has shown that some ICUs have implemented interventions and protocols that have been successful in decreasing CAUTI, and yet others who have done so have not been as fortunate. (Leblebicioglu et al., 2013). The previous method for preventing complications from installing a urinary catheter only delayed the infection process without preventing problems and without even giving prophylactic antibiotics (Cortese, 
Wagner, Tierney, Devine, \& Fogarty, 2018)This may eventually lead to the development of required national and international CAUTI prevention standards and protocols.

The implementation of preventative measures for CAUTI such as educational strategies, catheter avoidance, policies for catheter insertion, catheter selection, daily necessity review and limiting catheter days have been reported to be associated with decreased CAUTI rates in the ICU(Chenoweth \& Saint, 2013). The identification of the interventions with the greatest positive impact on CAUTI rates would be an asset to the healthcare professionals caring for patients with an in-dwelling catheter and for the clinic nurses developing policies. The aim of this study was to examine the existing evidence on the preventative interventions and protocols being implemented in ICUs and the impact that they have on the CAUTI rates and ultimately, patient outcomes.

\section{MATERIALS AND METHODS}

\section{Research Design}

A systematic review was the chosen research design, resulting from the latest research for the last 5 years. This study used the Preferred Reporting Items for Systematic Reviews and Meta-Analyzes (PRISMA) approach.

\section{Study Heterogeneity}

The process of searching for the articles was conducted electronically. The data was obtained from Scopus, Pubmed, EBSCO and Proquest. The literature review used the keywords 'prevention', 'CAUTI' and 'ICU'; "AND" was also used. After a number of articles was obtained, the researcher then selected them again according to the specified inclusion and exclusion criteria.

\section{Inclusion and Exclusion Criteria}

The desired articles were articles published between 2013 and 2018, and articles in English focusing on the selected keywords. The inclusion criteria involved databases which could reveal the effect of the CAUTI bundle on reducing the CAUTI incidence rate, various interventions that can reduce or prevent CAUTI events, actions that can have an effect on reducing CAUTI events, the steps or procedures that can prevent CAUTI occurrences, and the research sites namely the ICUs - where the studies were carried out. The exclusion criteria consisted of databases in the form of interventions that can reduce CAUTI but that use pharmacology, select research sites, where the patients studied are not in the ICU or where the patients do not have the same character as the patients in the ICU.

\section{Article Search Process}

The article search process was carried out between August and October 2018. The search for the articles was done by using the keywords that had been determined by the researchers and within the limits provided by the inclusion and exclusion criteria. The data obtained was then selected one by one by the researchers to determine the suitability of the articles. Duplicates were deleted. After obtaining the articles that were in accordance with what was set by the researchers, the articles were analyzed one by one and grouped to get the results. The next step was to discuss each intervention used to prevent the CAUTI.

\section{RESULTS}

\section{Study Selection}

The initial literature search returned 183 abstracts (39 from Scopus, 47 from Proquest, 13 from EBSCHOHOST and 84 from Pubmed). After reviewing the abstracts for relevance and matching them with the inclusion criteria, 31 articles were selected for a full-text review. There were 17 full-text articles excluded; 10 articles were not interventions and 7 articles were not set in the ICU. Finally, 14 articles were chosen to be reviewed, as listed in Figure 1.

\section{Study Heterogeneity}

The studies were heterogeneous. There were 6 cohort studies, 4 retrospective cohort studies, 3 interventional prospective studies and 1 quasiexperimental case control study. The duration of the CAUTI prevention strategy interventions in this study also varied, ranging from 5 months to several years. The age in this study began from 0 years to $>65$ years with an adult age being the average. The total respondents in this review were 42.486 patients with an average of 3,540 participants per study.

\section{Strategy of Prevention CAUTI in ICU}

From the 14 studies reviewed, all had an effects concerning reducing the incidence of infection in the urinary tract. There are several strategies used in terms of preventing CAUTI such as nursing rounds, CAUTI bundle, bacitracin, nurse-driven protocol, protocol by team / developmental protocol, surveillance of CAUTI, education, performance feedback, general culture or culturing practices within the ACCCM and IDSA guidelines. In this study, to reduce the incidence of CAUTI, there were 12 studies using a multi-dimensional approach strategy(Al-Hameed et al., 2018; Düzkaya, Bozkurt, Uysal, \& Yakut, 2016; Galiczewski, 2016; Galiczewski \& Shurpin, 2017; Gao et al., 2015; Kanj et al., 2013; Khamis et al., 2014; Leblebicioglu et al., 2013; Mullin et al., 2017; Navoa-Ng et al., 2013; Tyson et al., 2018; Underwood, 2015) and 2 other studies used a oneapproach strategy (Davies et al., 2018; Thomas, 2016)

\section{DISCUSSION}

Patients in the ICU are affected by critical conditions that are complex and that can change quickly. They are given treatment with invasive devices which reduces their immunity level. These factors make the 


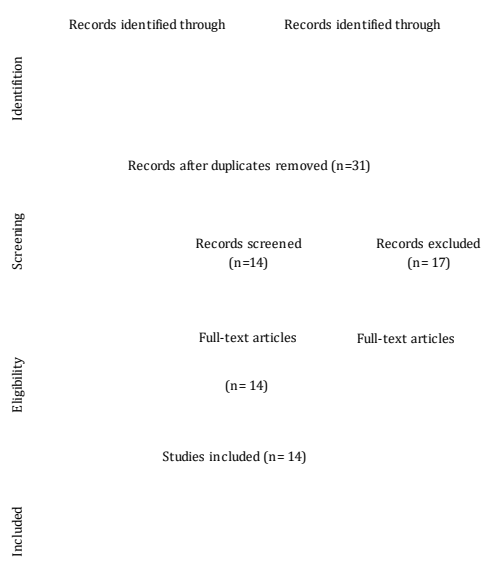

Figure 1. Flow diagram showing the selection of the studies

patients vulnerable to being infected, especially with opportunistic infections, in the hospital. Studies have reported that patients in ICUs are more likely to be infected than those in other departments (Gao et al., 2015). Urinary catheter insertion is a necessary and important procedure for patient care and management in ICU (Al-Hameed et al., 2018). The risk factors for the colonization of the periurethtral area by potential pathogens and CAUTI include invasive device insertion, catheterization time, severity of the underlying disease, the use of antibiotics, experience of the catheter insertion personnel, closed system problems, catheter blockage, renal dysfunction, and active infection in other body regions (Düzkaya et al., 2016).

Various prevention strategies have been suggested to decrease the incidence of CAUTIs in hospitalized patients. Improvements in urinary catheter aseptic insertion technique and maintenance practices have been shown to decrease the incidence of CAUTIs in multiple studies. Studies have shown that a bundle for preventing and controlling CAUTI could effectively decrease the incidence of CAUTI, which was decreased from $7.6 \%$ o to $5.0 \%$ o using such a method (Gao et al., 2015). Bundles for CAUTI are a set of standard measures that, when applied during the care of the patients, are confirmed to reduce the incidence of CAUTI. The advantage of the performance and checking of the elements of a bundle is that it acts a complete and consistent reminder system for the prevention of CAUTI (Khamis et al., 2014). The prevention bundle is focused on optimizing the technical aspects of urinary catheterization, including appropriate indications, insertion and maintenance techniques, and timely discontinuation, while concurrently standardizing urine culturing practices (Davies et al., 2018)

The International Nosocomial Infection Control Consortium (INICC) multi-dimensional infection control approach included the following items: (1) a bundle of infection control interventions; (2) education; (3) outcome surveillance; (4) process surveillance; (5) feedback on CAUTI rates and (6) performance feedback on the infection control practices (Leblebicioglu et al., 2013). The INICC multidimensional approach for controlling CAUTI included the following elements. First, an infection prevention bundle was implemented based on the guidelines published by SHEA and IDSA (Navoa-Ng et al., 2013), which provides evidence-based recommendations and cost-effective infection control measures that can be feasibly adapted to the ICU setting in developing countries. Second, the Health Care Workers (HCWs) were educated on infection preventive measures. Third, the CAUTI outcomes were monitored by applying the definitions for CAUTI as developed by the US CDC/NHSN (Navoa-Ng et al., 2013). Fourth, the CAUTI processes were monitored for compliance with easily measurable infection control measures, including hand hygiene performance. Fifth, feedback was provided on the CAUTI rates. Sixth, performance feedback was given on process surveillance, particularly by reviewing and discussing the chart results at the monthly infection control meetings.

Nurse-driven protocols generally require a physician's documentation for catheter criteria, including the specific need for prolonged catheterization. Appropriate indications for catheter use include acute urinary retention or bladder outlet obstruction, the need for accurate monitoring in critically ill patients, peri-operative catheter need following urologic or genitourinary surgery or for 24 hours after non-genitourinary surgery, for the protection of open sacral or perineal wounds in patients with urinary incontinence, prolonged immobilization due to unstable spine fractures or multiple pelvic fractures, or as an end-of-life comfort care measure. Urinary incontinence or prolonged postoperative catheterization with no other indications is an insufficient indication for catheterization. Under these protocols, nursing staff are encouraged to assess patients for the need for ongoing catheterization and to remove urinary catheters when they no longer meet the criteria (Thomas, 2016)

The nurse-driven protocols promoting early catheter removal in conjunction with improved catheter care and maintenance and improved urine culture ordering and collection practices can result in measurable decreases in both in-dwelling urinary catheter utilization and CAUTI rates in the STICU setting. In an era of quality improvement and patientcentered outcomes, this protocol represents a relatively low-cost resource which can serve as a useful adjunct for infection prevention in the STICU setting (Tyson et al., 2018).

Cranberry (Vaccinum macrocarpon) is thought to decrease the incidence of urinary tract infections through its many bioactive compounds. These include Type-A Proanthocyanins (PACs), fructose (known to decrease adhesions of fimbriated E-Coli to the bladder epithelium), ascorbic acid, hydroxybenzoic 
acid and favonols, which are known to exert antioxidant effects. It also contains ainulin, which is a prebiotic that enhances the growth of commensal EColi in the rectum. Vitamin $\mathrm{C}$ and Hippuric acid in the urine decrease urine $\mathrm{pH}$ and may act as a bacteriostatic agent, although its effectiveness is thought to be minimal. Bacitracin ointment, though active only against Gram-positive bacteria, may serve to prevent an ascending infection which plays an important role in the pathogenesis of recurrent UTI (Sorour et al., 2016). The addition of Cranberry Product (CP) and bacitracin metal care to the CDC CAUTI prevention bundle is associated with a reduction in CAUTI incidence. Cranberry Products have previously demonstrated promise in reducing UTI incidence. Two meta-analyses have evaluated CP in preventing non-catheter-associated UTI with mixed results. With respect to CAUTI, the use of CP in a single randomized controlled trial resulted in a reduction in CAUTI in women following gynecological surgery. Topical antibiotics seem to have an intuitive mechanism but they have been scarcely studied, and prior evidence has not supported routine use. Currently, the use of cranberry and topical bacitracin is not approved by the FDA for use in preventing urinary tract infections (Sorour et al., 2016).

\section{CONCLUSION}

The present review shows that there are several strategies that are used such as nursing rounds, CAUTI bundles, bacitracin and cranberry, nursedriven protocol, protocol through a team / developmental protocol, the surveillance of CAUTI, education, performance feedback and general cultural practices alongside the American College of Critical Care Medicine and the Infectious Disease Society of America guidelines, making up a multidimensional approach for the prevention and evaluation of incidences of CAUTI in ICU. A multidimensional approach is more used than a single dimensional approach in the ICU.

\section{REFERENCES}

Al-Hameed, F., Ahmed, G., AlSaedi, A., Bhutta, M., AlHameed, F., \& AlShamrani, M. (2018). Applying preventive measures leading to significant reduction of catheter-associated urinary tract infections in adult intensive care unit. Saudi Medical Journal, 39(1), 97-102. https://doi.org/10.15537/smj.2018.1.20999

Chenoweth, C., \& Saint, S. (2013). Preventing Catheter-Associated Urinary Tract Infections in the Intensive Care Unit. Critical Care Clinics, 29(1), 19-32. https://doi.org/10.1016/j.ccc.2012.10.005

Cortese, Y. J., Wagner, V. E., Tierney, M., Devine, D., \& Fogarty, A. (2018). Review of Catheter-Associated Urinary Tract Infections and In Vitro Urinary Tract Models. Journal of Healthare Engineering, 2018, 116.

Davies, P. E., Daley, M. J., Hecht, J., Hobbs, A., Burger,
C., Watkins, L., ... Brown, C. V. R. (2018). Effectiveness of a bundled approach to reduce urinary catheters and infection rates in trauma patients. American Journal of Infection Control, 46(7), 758-763. https://doi.org/10.1016/j.ajic.2017.11.032

Düzkaya, D. S., Bozkurt, G., Uysal, G., \& Yakut, T. (2016). The effects of bundles on catheterassociated urinary tract infections in the pediatric intensive care unit. Clinical Nurse Specialist, 30(6), 341-346.

https://doi.org/10.1097/NUR.00000000000002 46

Galiczewski, J. M. (2016). Interventions for the prevention of catheter associated urinary tract infections in intensive care units: An integrative review.

Galiczewski, J. M., \& Shurpin, K. M. (2017). An intervention to improve the catheter associated urinary tract infection rate in a medical intensive care unit: Direct observation of catheter insertion procedure. Intensive and Critical Care Nursing, 40, 26-34. https://doi.org/10.1016/j.iccn.2016.12.003

Gao, F., Wu, Y., Zou, J., Zhu, M., Zhang, J., Huang, H., \& Xiong, L. (2015). Impact of a bundle on prevention and control of healthcare associated infections in intensive care unit. Journal of Huazhong University of Science and Technology [Medical Sciences], 35(2), 283-290. https://doi.org/10.1007/s11596-015-1425-2

Gould, C. V, Umscheid, C. A., Agarwal, R. K., Kuntz, G., \& Pegues, D. A. (2017). Guideline for prevention of $C$ atheter - Associated Urinary Tract Infections 2009.

Kanj, S. S., Zahreddine, N., Rosenthal, V. D., Alamuddin, L., Kanafani, Z., \& Molaeb, B. (2013). Impact of a multidimensional infection control approach on catheter-associated urinary tract infection rates in an adult intensive care unit in Lebanon: International nosocomial infection control consortium (INICC) findings. International Journal of Infectious Diseases, 17(9), e686-e690. https://doi.org/10.1016/j.ijid.2013.01.020

Khamis, A. E., Amine, Omar, M., Helal, M., Mohamed, W., \& Bakr, K. (2014). Evaluation of an intervention program to prevent hospitalacquired catheter-associated urinary tract infections in an ICU in a rural Egypt hospital Evaluation eines Interventionsprogramms zur Prävention nosokomialer. GMS Hygiene and Infection Control, 9(2), 1-10.

Leblebicioglu, H., Ersoz, G., Rosenthal, V. D., NevzatYalcin, A., Akan, Ö. A., Sirmatel, F., ... Bacakoglu, F. (2013). Impact of a multidimensional infection control approach on catheter-associated urinary tract infection rates in adult intensive care units in 10 cities of Turkey: International Nosocomial Infection Control Consortium findings (INICC). American Journal of Infection Control, 41(10), 885891. https://doi.org/10.1016/j.ajic.2013.01.028

Mullin, K. M., Kovacs, C. S., Fatica, C., Einloth, C., 
Neuner, E. A., Guzman, J. A., ... Fraser, T. G. (2017). A multifaceted approach to reduction of catheterassociated urinary tract infections in the intensive care unit with an emphasis on stewardship of culturing. Infection Control and Hospital Epidemiology, 38(2), 186-188. https://doi.org/10.1017/ice.2016.266

Navoa-Ng, J. A., Berba, R., Rosenthal, V. D., Villanueva, V. D., Tolentino, M. C. V, Genuino, G. A. S., ... Mantaring, J. B. V. (2013). Impact of an International Nosocomial Infection Control Consortium multidimensional approach on catheter-associated urinary tract infections in adult intensive care units in the Philippines: International Nosocomial Infection Control Consortium (INICC) fi. Journal of Infection and Public Health, 6(5), 389-399. https://doi.org/10.1016/j.jiph.2013.03.002

Sorour, K., Care, C., Nuzzo, E., Resident, I. M., Tuttle, M., Chief, I. M., ... Medical, D. (2016). Addition of bacitracin and cranberry to standard Foley care reduces catheter-associated urinary tract infections. Canadian Journal of Infection Control, 31(3), 166-168.

Thomas, K. L. (2016). Reduction of CatheterAssociated Urinary Tract Infections Through the Algorithm and the Implementation of. J Wound Ostomy Continence Nurs, 43(April), 183-187. https://doi.org/10.1097/WON.00000000000002 06

Tyson, A. F., Campbell, E. F., Spangler, L. R., Ross, S. W., Reinke, C. E., Passaretti, C. L., \& Sing, R. F. (2018). Implementation of a Nurse-Driven Protocol for Catheter Removal to Decrease CatheterAssociated Urinary Tract Infection Rate in a Surgical Trauma ICU. Journal of Intensive Care Medicine, $\quad x x, \quad 1-7$. https://doi.org/10.1177/0885066618781304

Underwood, L. (2015). Series / Quality Performance Improvement The Effect of Implementing a Comprehensive Unit-Based Safety Program on Urinary Catheter Use. Urologic Nursing, 35(6), 271-279. https://doi.org/10.7257/1053- 